\title{
MORFOLOGÍA POLÍNICA DE LAS NYCTAGINACEAE NATIVAS DE ARGENTINA
}

\author{
Ezequiel E. Farrell' ${ }^{1}$ Lilia R. Mautino ${ }^{1,2}$ \& Silvina S. Garralla ${ }^{1,2}$
}

\begin{abstract}
${ }^{1}$ Centro de Ecología Aplicada del Litoral, Consejo Nacional de Investigaciones Cientificas y Técnicas y Universidad Nacional del Nordeste (CECOAL-CONICET-UNNE), Ruta 5, Km. 2,5, 3400 Corrientes, Argentina; ezequielfarrell@hotmail.com (autor corresponsal).

${ }^{2}$ Departamento de Biología, Facultad de Ciencias Exactas y Naturales y Agrimensura (FACENA), Universidad Nacional del Nordeste (UNNE). Avenida Libertad 5470, 3400 Corrientes, Argentina.
\end{abstract}

\begin{abstract}
Farrell, E. E.; L. R. Mautino \& S. S. Garralla. 2020. Pollen Morphology of natives Nyctaginaceae of Argentina. Darwiniana, nueva serie 8(1): 292-308.

The pollen morphology of Argentinian genera and species of Nyctaginaceae was analized in order to provide detailed information and contribute for their identification in actual and fossil pollen dispersed. The Nyctaginaceae family in Argentina, is composed of 8 genera with 19 species included in the following tribes: Boungainvilleeae: Bougainvillea (B. campanulata, B. infesta, B. praecox, B. spinosa, B. stipitata). Colignonieae: Colignonia (C. glomerata). Nyctagineae: Allionia (A. choisyi, A. incarnata), Boerhavia (B. cordobensis, B. diffusa, B. pulchella, B. spicata), Mirabilis (M. bracteosa, M. jalapa, M. ovata). Leucastereae: Reichenbachia (R. paraguayensis). Pisonieae: Pisonia (P. aculeata, P. zapallo), Pisoniella (P. glabrata). The pollen material was processed according to the Erdtman techniques, described and illustrated with light microscope and scanning electron microscope. The pollen grains are apolar or isopolar, radiosimetric; small to medium size, subcircular, subtriangular or cuadrangular in polar view, spheroidal, prolato-spheroidal, oblate-spheroidal or suboblate form. Tricolpate, tetracolpate, pantoporate or pantocolpoidate. Tectate perforate exine or semitectate reticulate. Variability found between genus, allows to differentiate them with the exception of Allionia and Mirabilis. In the genus represented with more than one species, Allionia, Boerhavia, Bougainvillea, and Mirabilis, it is not possible to differentiate the species from one another by the homogeneity and superposition of their characters, but it is possible in Pisonia. Keys for the identification are presented.
\end{abstract}

Keywords. Argentina; Nyctaginaceae; Pollen.

Resumen. Farrell, E. E.; L. R. Mautino \& S. S. Garralla. 2020. Morfología polínica de las Nyctaginaceae nativas de Argentina. Darwiniana, nueva serie 8(1): 292-308.

Se analiza la morfología del polen de los géneros y especies de Nyctaginaceae nativas de Argentina con el fin de brindar información detallada de ellos y aportar a su identificación en polen disperso actual y fósil. La familia Nyctaginaceae en Argentina, está integrada por 8 géneros con 19 especies, incluidas en las siguientes tribus: Boungainvilleeae: Bougainvillea (B. campanulata, B.infesta, B. praecox, B. spinosa, B. stipitata). Colignonieae: Colignonia (C. glomerata). Nyctagineae: Allionia (A. choisyi, A. incarnata), Boerhavia (B. cordobensis, B. diffusa, B. pulchella, B. spicata), Mirabilis (M. bracteosa, M. jalapa, M. ovata). Leucastereae: Reichenbachia (R. paraguayensis). Pisonieae: Pisonia (P. aculeata, P. zapallo), Pisoniella ( $P$. glabrata). El material polínico fue procesado según la técnica de Erdtman, descripto e ilustrado con imágenes obtenidas con microscopio óptico y electrónico de barrido. Como resultado se determinó que las especies argentinas de Nyctaginaceae son apolares o isopolares, radiosimétricos, de tamaño pequeño a muy grande, ámbito subcircular, subtriangular o cuadrangular, forma esferoidal, prolato esferoidal, oblato esferoidal o sub-oblata. Con aperturas simples (tricolpados, tetracolpados, pantoporados o pantocolpoidados). Exina tectada perforada o semitectada reticulada. La variabilidad encontrada a nivel de género permite diferenciarlos, para lo cual se presentan claves de identificación con la excepción de Allionia y Mirabilis. En los géneros representados con más de una especie, Allionia, Boerhavia, Bougainvillea, y Mirabilis, no pueden diferenciarse palinológicamente las especies entre sí por la homogeneidad y superposición de sus caracteres, no obstante, es posible en Pisonia.

Palabras clave. Argentina; Nyctaginaceae; Polen. 


\section{INTRODUCCIÓN}

La familia Nyctaginaceae Juss. reúne 405 especies en 31 géneros distribuidos en las regiones tropicales, subtropicales y templadas del mundo (APG IV, 2016). En Argentina se citan 8 géneros y 19 especies (Mabberley, 1987; Zuloaga \& Morrone, 1999; Zuloaga et al., 2008) incluidas en las siguientes tribus según Douglas \& Spellenberg (2010): Tribu Bougainvilleeae Choisy: Bougainvillea campanulata Heimerl, Bougainvillea infesta Griseb., Bougainvillea praecox Griseb., Bougainvillea spinosa (Cav.) Heimerl y Bougainvillea stipitata Griseb.; Tribu Colignonieae Standl: Colignonia glomerata Griseb.; Tribu Leucastereae Benth. y Hook.: Reichenbachia paraguayensis (D. Parodi) Dugand \& Daniel; Tribu Nyctagineae Horan.: Allionia choisyi Standl., Allionia incarnata L., Boerhavia cordobensis Kuntze, Boerhavia diffusa L., Boerhavia pulchella Griseb., Boerhavia spicata Choisy, Mirabilis bracteosa (Griseb.) Heimerl, Mirabilis jalapa L. y Mirabilis ovata (Ruiz \& Pav.) F. Meigen; Tribu Pisonieae Meisn.: Pisonia aculeata L., Pisonia zapallo Griseb. y Pisoniella glabrata (Heimerl) Standl.

Los antecedentes de estudios palinológicos de las especies argentinas son escasos e incompletos. Entre ellos se destaca el trabajo de Caccavari (1979), quién analizó sólo al microscopio óptico 9 géneros, 16 especies y 3 variedades $(A$. incarnata, Boerhavia coccinea Mill., B. pulchela, Bougainvillea campanulata, B. infesta, B. praecox, B. spinosa, B. stipitata, C. glomerata, M. bracteosa var. bracteosa, M. bracteosa var. micrantha Toursark., M. jalapa, Oxybaphus ovatus (Ruiz \& Pav.) Vahl, P. aculeata, P. zapallo var. zapallo, P. zapallo var. guaranitica Toursark, Pisoniella arborescens var. glabra Heimerl y Reichenbachia hirsuta Spreng.), presentando una clave polínica para su identificación. Anteriormente, Markgraf \& D'Antoni (1978) describieron también solo al microscopio óptico 5 especies argentinas (Allionia incarnata, Boerhavia paniculata Rich., Bougainvillea spinosa, Oxybaphus ovatus y Pisonia ambigua Heimerl). Por último, Farrell \& Mautino (2018) presentan los resultados preliminares de este estudio. De igual modo el polen de algunas especies de Nyctaginaceae procedentes de otros países también fue estudiado por Erdtman, 1966; Nowicke, 1970; Nowicke \& Luikart 1971; Barth \& Ferreira Barbosa, 1972; Kannabiran, 1973; Sánchez-Dzib et al., 2009; Albuquerque de Souza et al., 2010; Tripathi et al., 2017 y Xu \& Deng, 2017.

$\mathrm{Al}$ analizar las publicaciones paleopalinológicas del Neógeno de los últimos veinte años para Argentina, Farrell (2017) logró identificar polen fósil afín a Nyctaginaceae en diferentes formaciones geológicas, señalando la representación de la citada familia en el registro fósil del país.

Por otro lado, actualmente los trabajos melisopalinológicos se encuentran en mayor auge, ya que permiten caracterizar las mieles de acuerdo con su contenido polínico, origen botánico y geográfico; sirven también como medio de control de calidad y para evitar el fraude comercial (Rodríguez de la Cruz et al., 2013; Ramírez Arriaga et al., 2016). Si bien el contenido de polen de Nyctaginaceae en mieles para el país se encuentra poco representado, esta podría deberse a la dificultad para la identificación del polen.

Por lo tanto, el presente trabajo, tiene por objetivo caracterizar palinológicamente las especies nativas de Nyctaginaceae de Argentina, comparar los datos obtenidos con otras publicaciones a fin de enriquecer el rango de variación de los caracteres conocidos y contribuir a la identificación y determinación del polen disperso contenido en mieles y en sedimentos fósiles y actuales, entre otros.

\section{MATERIALES Y MÉTODOS}

El material polínico fue obtenido del herbario del Instituto de Botánica del Nordeste (CTES) $\mathrm{y}$ del herbario del Museo Argentino de Ciencias Naturales (BA). Los botones florales fueron procesados en el laboratorio de palinología del CECOAL (CONICET-UNNE) de acuerdo con la técnica de Acetólisis de Erdtman (1966). Se utilizó glicerina gelatina como medio de montaje para su observación al microscopio óptico (MO). Los granos acetolizados se deshidrataron en series crecientes de alcoholes $(50,70,90$ y $100 \%$ ) y se metalizaron con oro-paladio para su posterior observación al microscopio electrónico de barrido (MEB). Los preparados palinológicos obtenidos fueron incorporados a la Palinoteca de 
la Universidad Nacional del Nordeste (CTESPAL). Las observaciones, interpretaciones y fotomicrografías se realizaron con Microscopio óptico compuesto trinocular Nykon ECLIPSE E 100 equipado con cámara digital Canon Power Shot 550 y al MEB se efectuaron con el equipo JEOL/ JSM- 580 OLV (UNNE) y JEOL 6480 LV (UNSA).

De cada especie se midieron como mínimo 30 granos y la terminología adoptada es la sugerida por Erdtman \& Straka (1961), Erdtman (1966), Kremp (1968), Nilsson \& Praglowski (1992), Sáenz Laín (2004) y Punt et al. (2007).

\section{Material examinado}

Allionia choisyi: ARGENTINA. La Rioja: Depto. Capital, Pensiero et al. 7474 (CTES), PALCTES 7271. Salta: Depto. Cach, Kravovickas et al. 21935 (CTES), PAL-CTES 7272.

Allionia incarnata: ARGENTINA. Jujuy: Depto. Yaví, Pauta y Souza 7646 (CTES), PALCTES 7273. Catamarca: Depto. Chamical, Leguiza y Carrizo 64 (CTES), PAL-CTES 7274. La Rioja: Depto. Capital, Biurrum y Agüero 8268 (CTES), PAL-CTES 7283. Salta: Depto. Metán, Zuloaga et al. 10079 (CTES), PAL-CTES 7284.

Boerhavia cordobensis: ARGENTINA. La Rioja: Depto. Gral. San Martín, Pedersen 15195 (CTES), PAL-CTES 7277. San Juan: Depto. Caucete, Pensiero et al. 7377 (CTES), PAL-CTES 7278.

Boerhavia diffusa var. diffusa: ARGENTINA. Misiones: Depto. Iguazú, Gatti 12 (CTES), PALCTES 7190. Corrientes: Depto. Capital, Carnevali 483 (CTES), PAL-CTES 7191. Boerhavia diffusa var. leiocarpa: ARGENTINA. Salta: Depto. Rivadavia, Scarpa 500 (CTES), PAL-CTES 7192. PARAGUAY. Boquerón: Perez et al. 2819 (CTES), PAL-CTES 7193.

Boerhavia pulchella: ARGENTINA. Córdoba: Depto. Punilla, Cosquín, Rua 18093 (CTES), PALCTES 7276. San Juan: Depto. Jáchal, Donadio et al. 48 (CTES), PAL-CTES 7279. Santiago del Estero: Depto. Ojo de Agua, Sierra de Sumampa, Pedersen 15156 (CTES), PAL-CTES 7280.

Boerhavia spicata: ARGENTINA. Catamarca: Depto. Tinogasta, Pedersen 15318 (CTES), PALCTES 7275.

Bougainvillea campanulata: ARGENTINA. Jujuy: Depto. San Pedro, Sta. Clara, Schreiter
1255 (BA), PAL CTES 7250; San Pedro, Schreiter 1257 (BA), PAL-CTES 7251. Tucumán: Loc. Vipos, Capurro 56278 (BA), PAL-CTES 7252; Depto. Trancas, Venturi 393 (BA), PAL-CTES 7249. Jujuy: Depto. Santa Barbara, Cabrera 16066 (CTES), PAL-CTES 7194. Salta: Depto. Güemes, Saravia Toledo 1726 (CTES), PAL-CTES 7227. Formosa: Depto. Matacos, Ing. Juárez, Juárez 840 (CTES), PAL-CTES 7213.

Bougainvillea infesta: ARGENTINA. Salta: El Carmen, Schinini 19531 (CTES), PAL-CTES 7197; Chuquiasca, Nelsen 10222 (CTES), PALCTES 7198.

Bougainvillea praecox: ARGENTINA. Salta: Depto. Metán, Pedersen 15864 (CTES), PALCTES 7200. Chaco: Loc. Miraflores, Salgado 448 (CTES), PAL-CTES 7214.

Bougainvillea spinosa: ARGENTINA. Mendoza: Depto. Malargüe, Prina et al. 2562 (CTES), PAL-CTES 7264. Jujuy: Depto. Humahuaca, Zuloaga et al. 5956 (CTES), PALCTES 7265. Salta: Loc. Lerma, Dematteis $y$ Schinini 2680 (CTES), PAL-CTES 7281.

Bougainvillea stipitata: ARGENTINA. Jujuy: Depto. Valle Grande, Morrone et al. 3665 (CTES), PAL-CTES 7201. Catamarca, Menéndez et al. 9394 (CTES), PAL-CTES 7202.

Colignonia glomerata: ARGENTINA. Tucumán, Schultz 8482 (CTES), PAL-CTES 7266. Jujuy: Depto. Capital, Fabris 3929 (CTES), PALCTES 7267. Salta, Depto. Capital, San Lorenzo, Novara 10966 (CTES), PAL-CTES 7284.

Mirabilis bracteosa var. micrantha: ARGENTINA. Jujuy: Depto. Capital, Cocucci et al. 2650 (CTES), PAL-CTES 7268.

Mirabilis jalapa: ARGENTINA. Corrientes: Depto. Paso de los Libres, Lourteig et al. 2716 (CTES), PAL-CTES 7203; Depto. Esquina, Krapovickas et al. 26917 (CTES), PAL.CTES 7204. Misiones: Depto. El Dorado; Keller 618 (CTES), PAL-CTES 7219. Mirabilis ovata: ARGENTINA. San Juan: Depto. Jachal; Zavala et al. 45 (CTES), PAL-CTES 7206. Jujuy: Pob. Tilcara, Garganta del Diablo, Fabris et al. 6035 (CTES), PAL-CTES 7205.

Pisonia aculeata: ARGENTINA. Chaco: Loc. Antequera, Schinini 8558 (CTES), PAL-CTES 7207. Corrientes: Depto. Capital, Pueyo 4768 (CTES), PAL-CTES 7216. 
Pisonia zapallo: ARGENTINA. Misiones: Munic. Guarani, Tressens et al. 5668 (CTES), PAL-CTES 7218. Formosa: Depto. Bermejo, Arenas 3484 (CTES), PAL-CTES 7208.

Pisoniella glabrata: ARGENTINA. Tucumán: Schultz 6539(CTES), PAL-CTES 7269. Salta: Depto. Rosario de Lerma, Quebrada del Toro, Ruta No $51 \mathrm{~km}$. 38, Novara 11920 (CTES), PAL-CTES 7270.

Reichenbachia paraguayensis: PARAGUAY. Carapeguá: Mereles 8484 (CTES), PAL-CTES 7209. Asunción: Schinini 24091 (CTES), PALCTES 7210.

\section{RESULTADOS}

\section{Descripción general}

Los granos de polen de las especies de Nyctaginaceae de Argentina son apolares o isopolares, radiosimétricos, de tamaño pequeño a muy grande (de 16 a $200 \mu \mathrm{m}$ ), ámbito subcircular, subtriangular o cuadrangular, la forma es esferoidal, prolata-esferoidal, oblata-esferoidal o sub-oblata. Con aperturas simples (tricolpados, tetracolpados, pantoporados o pantocolpoidados). Exina tectada perforada o semitectada reticulada de espesor uniforme en todo el grano y clara diferenciación entre sexina y nexina con columelas simples. Los granos con exina tectada perforada poseen espinas y/o microespinas supratectales, con perforaciones con o sin engrosamiento anular. Los granos semitectados, son reticulados, heterobrochados con microespinas supratectales; lúmenes con columelas y gránulos libres o sin ellos. Membranas aperturales lisas u ornamentadas con gránulos y microespinas.

Se presentan las descripciones polínicas de las especies analizadas agrupadas por tribus a las que pertenecen.

\section{Tribu Bougainvilleeae (Figs. 1 y 2) \\ Género Bougainvillea}

Granos isopolares, radiosimétricos, de tamaño mediano, ámbito subcircular, oblato-esferoidales y sub-oblatos, tri y tetracolpados. Exina semitectada, reticulada, heterobrochada, latimurada, lúmenes de forma variable con elementos positivos libres, algunas columelas fusionadas. Con microespinas supratectales.
Los valores del eje polar, diámetro ecuatorial (mínimo-máximo), P/E, espesor de la exina, sexina y nexina de cada una de las especies del género se expresan en la Tabla 1.

\section{Bougainvillea campanulata (Fig. 1 A-D)}

Granos oblato-esferoidales, tetracolpados (98\%) y tricolpados (2\%). Colpos de 8-14,5 $\mu \mathrm{m}$ de largo, mesocolpio de $12-24 \mu \mathrm{m}$ y apocolpio de 7-24,5 $\mu \mathrm{m}$. Lúmenes con elementos libres o sin ellos. Cuando presentes los elementos positivos libres son gránulos y columelas.

MEB: Los lúmenes miden 1 a $5 \mu \mathrm{m}$ de diámetro, aquellos con elementos libres tienen 2 a 8 gránulos de $\pm 0,5 \mu \mathrm{m}$ y escasas columelas de $\pm 0,8 \mu \mathrm{m}$ de alto. Muros de $\pm 0,8 \mu \mathrm{m}$ de ancho, con microespinas supratectales $\leq 0,4 \mu \mathrm{m}$ de alto. Columelas simples de $\pm 0,5 \mu \mathrm{m}$ de alto.

\section{Bougainvillea infesta (Fig. 1 E-H)}

Granos oblato-esferoidales, tricolpados (tetracolpados $<$ de 1\%). Colpos de 11-17,5 $\mu \mathrm{m}$ de largo, mesocolpio de 22-27 $\mu \mathrm{m}$ y apocolpio de 17-28 $\mu \mathrm{m}$. Lúmenes con 2-8 elementos positivos libres de diferentes alturas, algunos de ellos tienen la misma altura de las columelas.

MEB: Lúmenes de 2 a $6,5 \mu \mathrm{m}$ de diámetro, algunos con columelas de $\pm 0,6 \mu \mathrm{m}$ de alto y gránulos de $\pm 0,3 \mu \mathrm{m}$ libres en número de hasta 12 elementos por lumen. Los muros de $\pm 0,7 \mu \mathrm{m}$ de ancho, con microespinas supratectales de $\pm 0,2 \mu \mathrm{m}$ de alto. Columelas simples de $0,5 \mu \mathrm{m}$ de alto.

Bougainvillea praecox (Fig. 1 I-L)

Granos oblato-esferoidales, tricolpados (97\%) y tetracolpados (3\%). Colpos de 7-15 $\mu \mathrm{m}$ de largo, mesocolpio de $17 \mu \mathrm{m}$ y apocolpio de $15-28$ $\mu \mathrm{m}$. Lúmenes con elementos positivos libres de diferentes alturas.

MEB: Lúmenes de 0,8 a $3,8 \mu \mathrm{m}$ de diámetro, con 1 a 6 gránulos de 0,3-0,9 $\mu$ m. Muros de $\pm 0,8$ $\mu \mathrm{m}$ de ancho con microespinas supratectales $\leq 0,4$ $\mu \mathrm{m}$ de alto. Columelas simples de $0,6 \mu \mathrm{m}$ de alto.

\section{Bougainvillea spinosa (Fig. 1 M-P)}

Granos oblato-esferoidales, tricolpados (95\%), tetracolpados (5\%). Colpos de 5-20 $\mu \mathrm{m}$ de largo, mesocolpio de 15-33 $\mu \mathrm{m}$ y apocolpio 8-33 $\mu \mathrm{m}$. Lúmenes con 1 a 15 elementos positivos libres. 

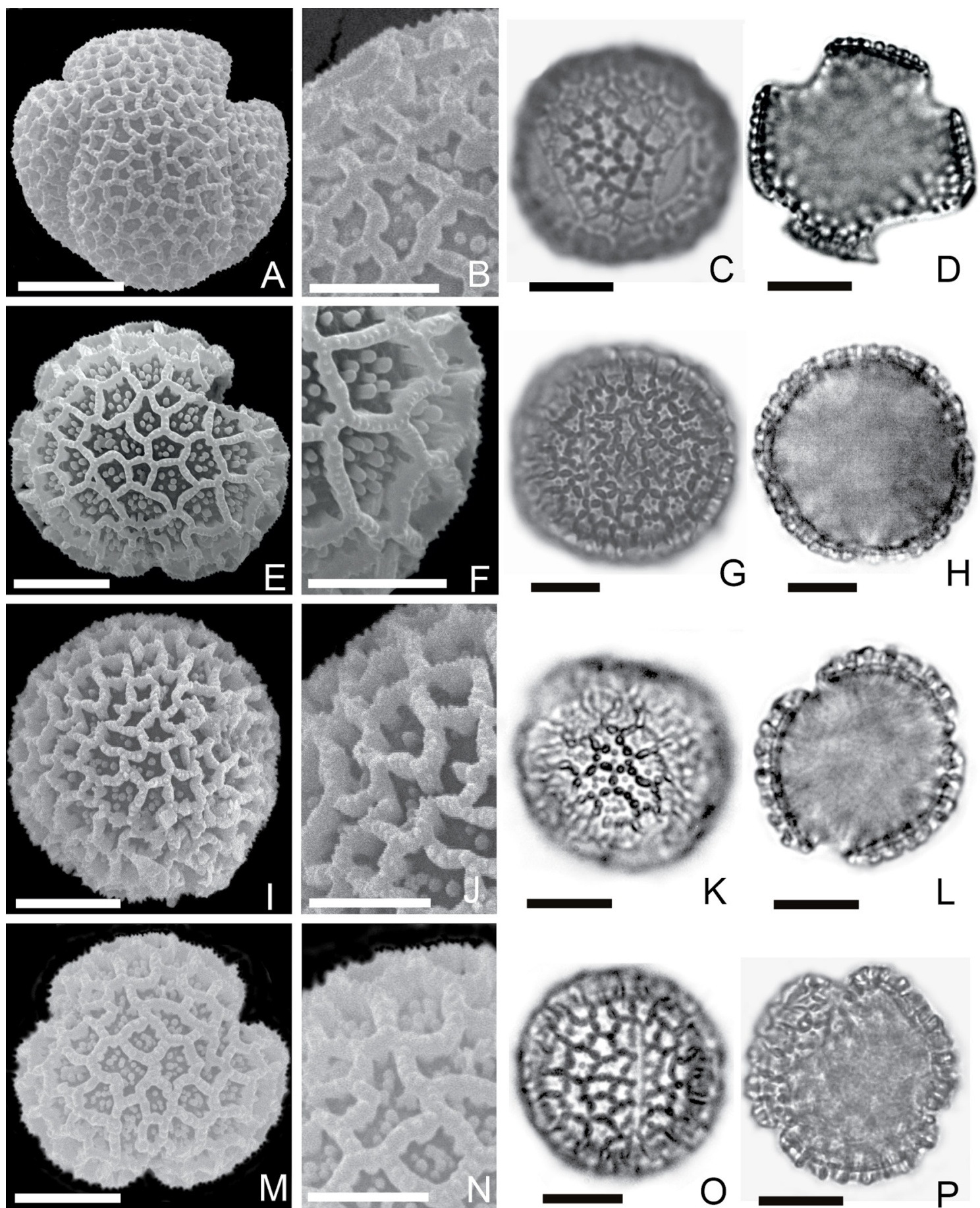

Fig. 1. Bougainvillea campanulata. A, vista subecuatorial (MEB). B, detalle de la exina (MEB). C, vista ecuatorial (MO). D, vista polar en corte óptico (MO). Bougainvillea infesta. E, vista polar (MEB). F, detalle de la exina (MEB). G, vista ecuatorial (MO). H, vista polar en corte óptico (MO). Bougainvillea praecox. I, vista polar (MEB). J, detalle de la exina (MEB). K, vista polar (MO). L, vista polar en corte óptico (MO). Bougainvillea spinosa. M, vista polar (MEB). $\mathbf{N}$, detalle de la exina (MEB). O, vista ecuatorial (MO). P, vista polar en corte óptico (MO). La escala equivale a $10 \mu \mathrm{m}$. 
Tabla 1. Valores del (P) eje polar, (E) diámetro ecuatorial (mínimo - máximo), P/E, espesor de la exina, nexina y sexina de las especies del género Bougainvillea.

\begin{tabular}{|l|c|c|c|c|c|c|}
\hline \multicolumn{1}{|c|}{ Especie } & $\mathrm{P}(\mu \mathrm{m})$ & $\mathrm{E}(\mu \mathrm{m})$ & $\mathrm{P} / \mathrm{E}$ & Exina $(\mu \mathrm{m})$ & Nexina $(\mu \mathrm{m})$ & Sexina $(\mu \mathrm{m})$ \\
\hline Bougainvillea campanulata & $20-29$ & $24-32$ & 0,9 & $1,5-3$ & $0,5-1$ & $1-2$ \\
\hline Bougainvillea infesta & $27-33$ & $31-33$ & 0,9 & $2,5-3$ & $0,5-1$ & $2-3$ \\
\hline Bougainvillea praecox & $22-27$ & $25-32$ & 0,9 & $2,5-3,5$ & $0,5-1,5$ & $2-3$ \\
\hline Bougainvillea spinosa & $15-46$ & $21-44$ & 0,9 & $1,5-3,5$ & $0,5-1$ & $1-2,5$ \\
\hline Bougainvillea stipitata & $22-28$ & $26-32$ & 0,8 & $2,7-4$ & $0,7-1$ & $2-3$ \\
\hline
\end{tabular}

MEB: Lúmenes de 1,7 a $5 \mu \mathrm{m}$ de diámetro con columelas libres $\leq 0,5 \mu \mathrm{m}$ y escasos gránulos de \pm $0,4 \mu \mathrm{m}$ algunos de estos se presentan fusionados. Muros de $\pm 0,7 \mu \mathrm{m}$ de ancho con microespinas supratectales de $\pm 0,5 \mu \mathrm{m}$ de alto. Columelas simples de $0,5 \mu \mathrm{m}$ de alto.

\section{Bougainvillea stipitata (Fig. 2 A-D)}

Granos sub-oblatos, tricolpados. Colpos de 7-17 $\mu \mathrm{m}$ de largo, mesocolpio de 20-26 $\mu \mathrm{m}$ y apocolpio de 15-21 $\mu \mathrm{m}$. Lúmenes con hasta 9 elementos positivos libres de diferentes alturas, algunos llegan a tener la altura de las columelas.

MEB: Lúmenes de 2 a $7 \mu \mathrm{m}$ de diámetro con columelas libres de $\pm 1 \mu \mathrm{m}$ de alto y escasos gránulos $<0,5 \mu \mathrm{m}$. Muros de $\pm 1 \mu \mathrm{m}$ de ancho con microespinas supratectales de $\pm 0,3 \mu \mathrm{m}$ de alto. Columelas simples de 0,9-1,5 $\mu \mathrm{m}$ de alto.

\section{Tribu Leucastereae (Fig. 2) \\ Género Reichenbachia}

Granos isopolares, radiosimétricos, de tamaño pequeño, ámbito subcircular, oblatoesferoidales, tricolpados. Exina semitectada, reticulada, heterobrochada, latimurada, lúmenes de forma variable. Con microespinas supratectales.

Reichenbachia paraguayensis (Fig. 2 E-H)

$\mathrm{P}=16-21 \mu \mathrm{m} ; \mathrm{E}=18-22 \mu \mathrm{m} ; \mathrm{P} / \mathrm{E}=0,9$. Colpos de 7-12 $\mu \mathrm{m}$ de largo. Mesocolpio de 10-18 $\mu \mathrm{m}$ y apocolpio de 11-18 $\mu \mathrm{m}$. Sexina de 0,7-2,5 $\mu \mathrm{m}$ y nexina de $\pm 0,7 \mu \mathrm{m}$ de espesor.

MEB: Lúmenes de 0,5-2,5 $\mu \mathrm{m}$ de diámetro, muros de $\pm 0,6 \mu \mathrm{m}$ de ancho con microespinas supratectales de $\pm 0,2 \mu \mathrm{m}$ de alto. Columelas de $\pm 0,5 \mu \mathrm{m}$ de alto.
Tribu Colignonieae (Figs. 2 y 6 )

\section{Género Colignonia}

Granos apolares, radiosimétricos, de tamaño mediano, esferoidales, pantoporados, exina tectada con microespinas y perforaciones.

Colignonia glomerata (Fig. 2 I-K y Fig. 6 B)

Granos de 21 a $44 \mu \mathrm{m}$ de diámetro con 12 poros circulares a subcirculares, de 3-8 $\mu \mathrm{m}$ de diámetro con membrana apertural esculturada. Los poros siguen una distribución regular, como si se dispusieran en los vértices de un cuboctaedro (ver esquema representativo). Exina escabrada de $\pm 3 \mu \mathrm{m}$. Sexina de $\pm 2 \mu \mathrm{m}$ y nexina de $\pm 1 \mu \mathrm{m}$.

MEB: Tectum con microespinas de $\pm 0,4 \mu \mathrm{m}$ de alto y perforaciones $\leq 0,4 \mu \mathrm{m}$ de diámetro.

\section{Tribu Nyctagineae (Figs. 3 y 4 )}

Los valores del diámetro de los granos (mínimo (promedio) máximo), del número de poros, diámetro de los poros (mínimo-máximo) y del espesor de la exina, sexina y nexina de los géneros y especies argentinas de esta tribu se expresan en la Tabla 2.

\section{Género Allionia}

Granos apolares, radiosimétricos, de tamaño grande, esferoidales, pantoporados. Poros subcirculares. Exina tectada, equinada, microequinada, perforaciones con engrosamiento anular. Elementos esculturales distribuidos al azar, con nexina mayor que la sexina.

Allionia choisyi (Fig. 3 A-D)

Granos con poros con membrana apertural que portan 1-2 elementos positivos menores de $1 \mu \mathrm{m}$. 

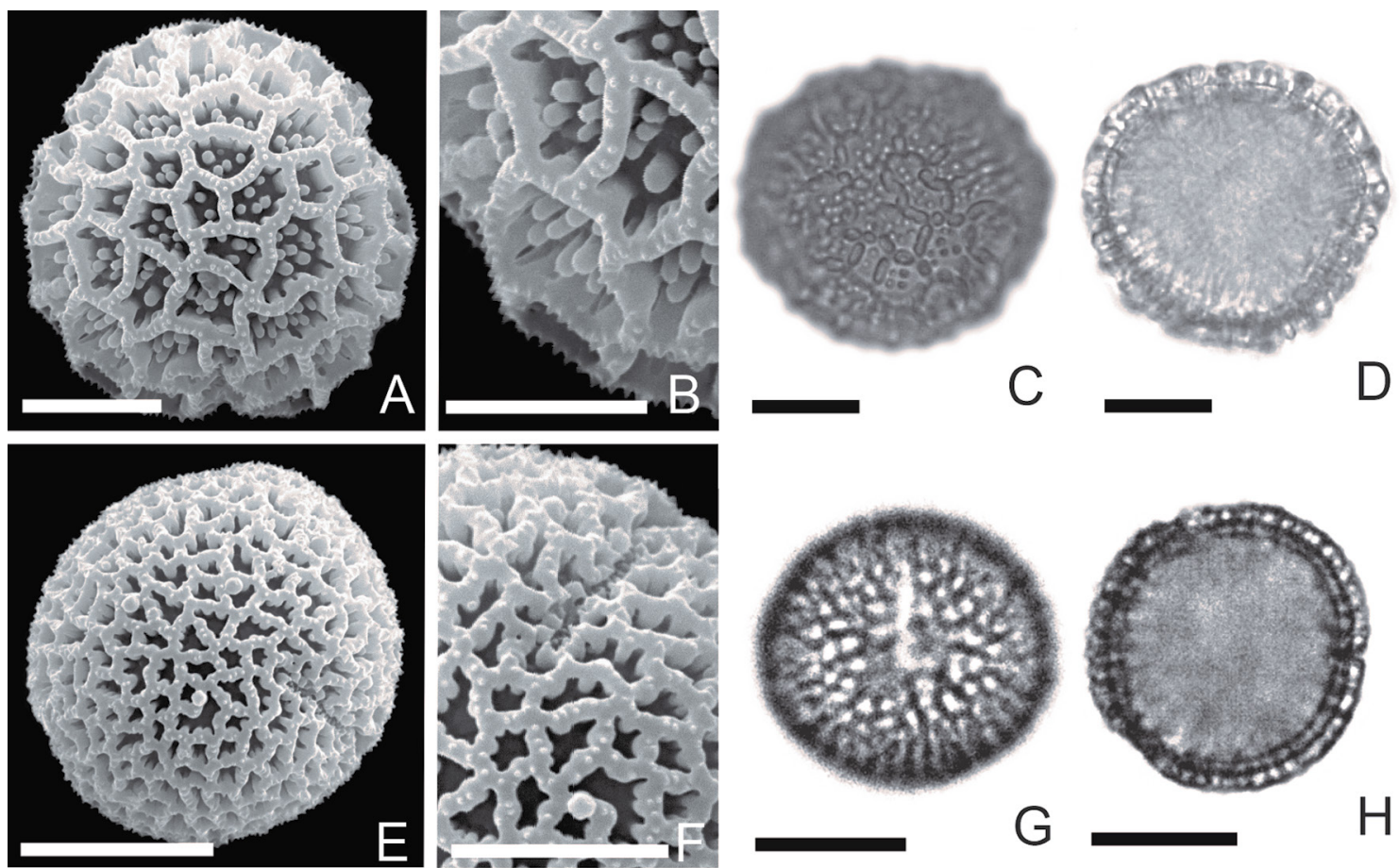

D
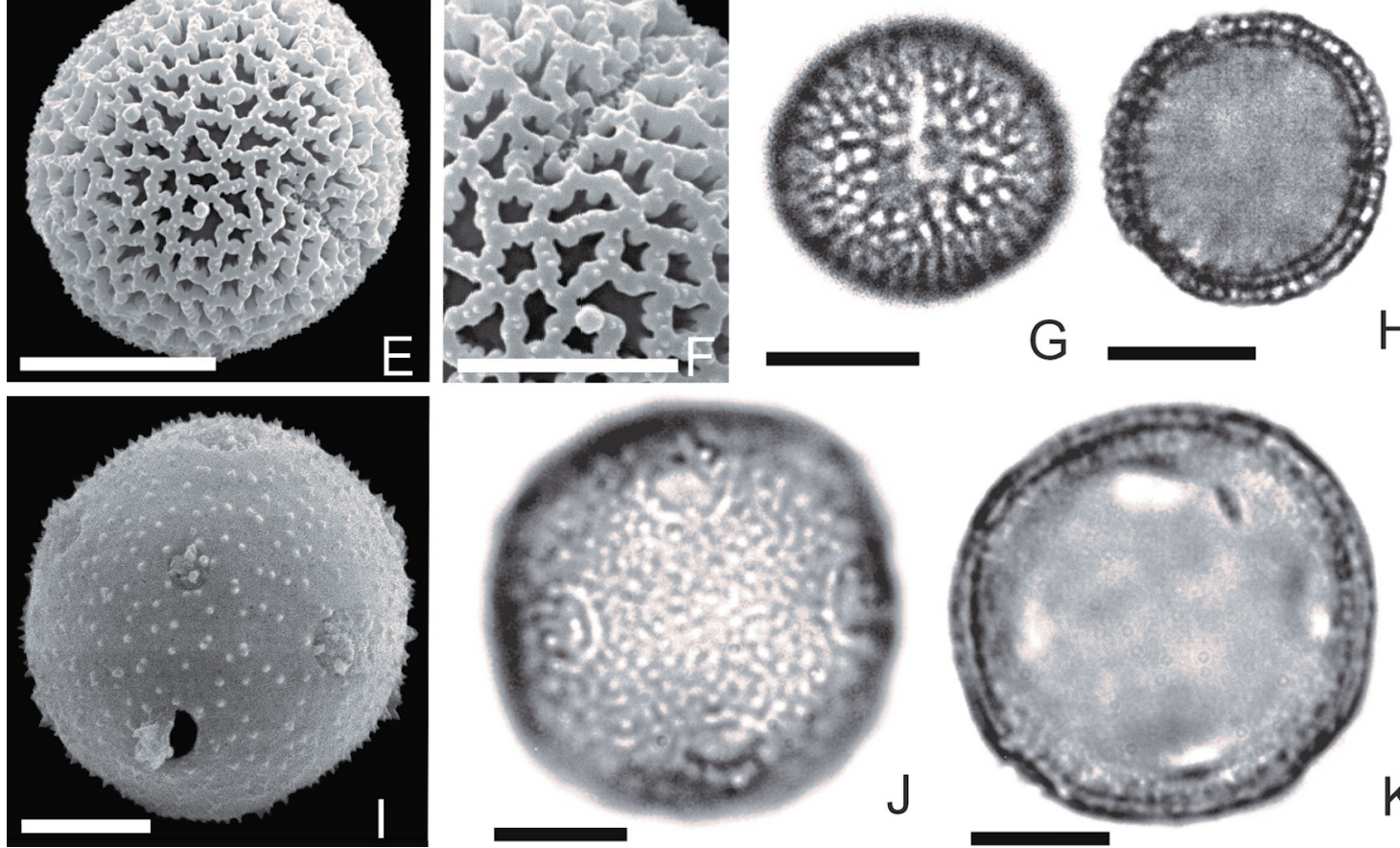

G

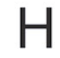

Fig. 2. Bougainvillea stipitata. A, vista polar (MEB). B, detalle de la exina (MEB). C, vista polar (MO). D, vista polar en corte óptico (MO). Reichenbachia paraguayensis. E, vista polar (MEB). F, detalle de la exina (MEB). G, vista ecuatorial (MO). H, vista polar en corte óptico (MO). Colignonia glomerata. I, vista general (MEB). $\mathbf{J}$, foco superior (MO). K, corte óptico (MO). La escala equivale a $10 \mu \mathrm{m}$.

Tectum equinado y microequinado, las microespinas presentan engrosamiento en su base. Columelas $<1 \mu \mathrm{m}$ de alto.

MEB: Tectum con microespinas y espinas $<2,5 \mu \mathrm{m}$ de alto y de $\pm 2 \mu \mathrm{m}$ en la base, perforaciones rodeadas de un engrosamiento anular de 0,7-2 $\mu \mathrm{m}$ de diámetro total. Membranas aperturales con espinas de $\pm 1,5 \mu \mathrm{m}$ y gránulos $\leq 0,5 \mu \mathrm{m}$.
Allionia incarnata (Fig. 3 E-H)

Granos con poros con membrana apertural portando una microespina sobre ella. El tectum presenta microespinas y otros elementos positivos bajos. Columelas $<1 \mu \mathrm{m}$ de alto.

MEB: Tectum con espinas de 1-3 $\mu \mathrm{m}$ y perforaciones rodeadas de un engrosamiento anular de 0,7-3 $\mu \mathrm{m}$ de diámetro total. Membranas aperturales con espinas de $\pm 2 \mu \mathrm{m}$. 

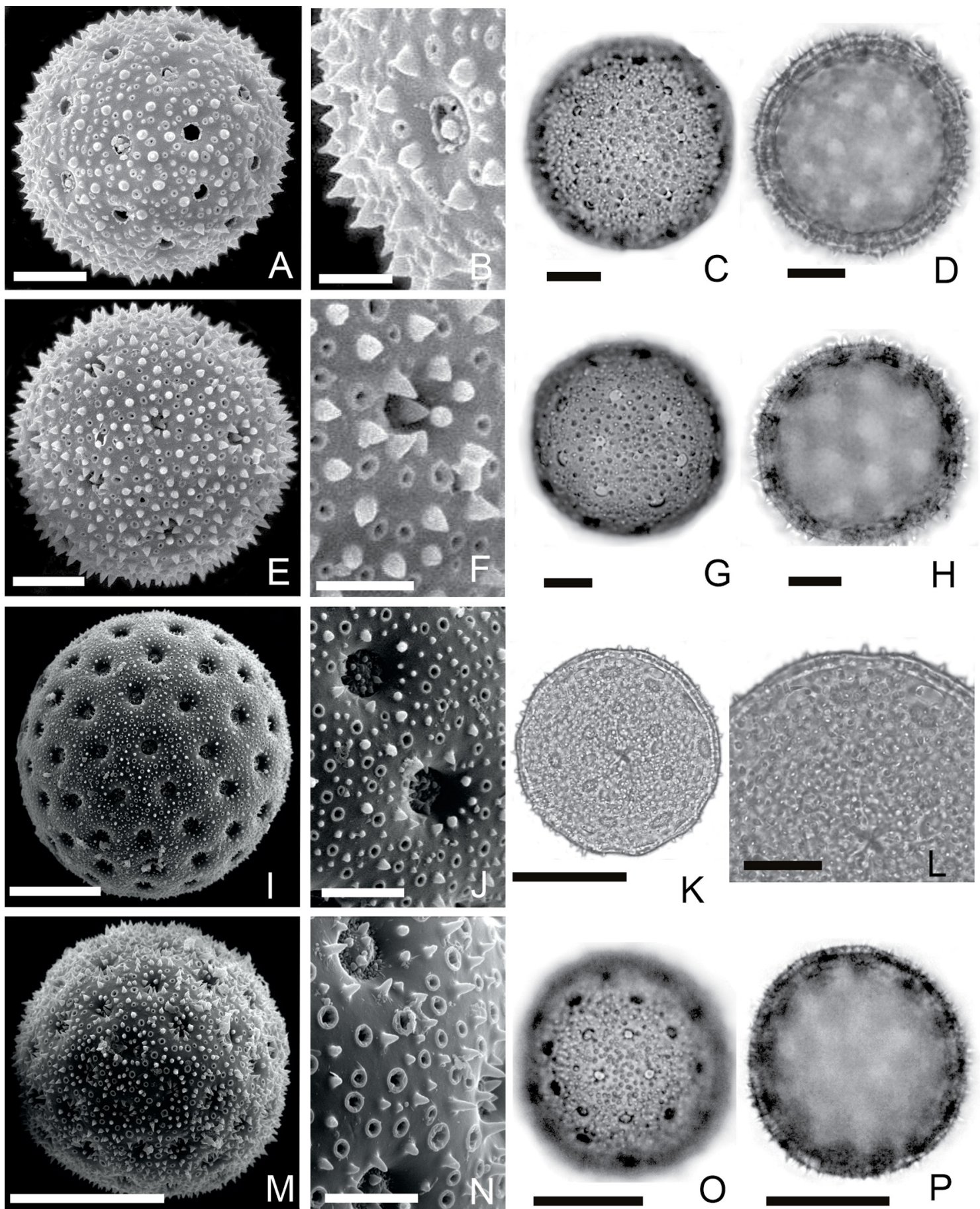

Fig. 3. Allionia choisyi. A, vista general (MEB). B, detalle de la exina y poro (MEB). C, vista general (MO). D, corte óptico (MO). Allionia incarnata. E, vista general (MEB). F, detalle de la exina y poro (MEB). G, vista general (MO). H, corte óptico (MO). Mirabilis jalapa. I, vista general (MEB). J, detalle de la exina y poro (MEB). Mirabilis bracteosa. K, vista general (MO). L, detalle de la exina y poros (MO). Mirabilis ovata. M, vista general (MEB). $\mathbf{N}$, detalle de la exina y poro (MEB). $\mathbf{O}$, vista general (MO). $\mathbf{P}$, corte óptico (MO). La escala equivale a $20 \mu \mathrm{m}$ en A, C, D, E, G, H, I, K, L, M, O, P y a $5 \mu \mathrm{m}$ en B, F, J, N. 
Tabla 2. Valores del diámetro de los granos (mínimo (promedio) máximo), del número de poros, diámetro de los poros (mínimo-máximo) y del espesor de la exina, sexina y nexina de las especies de los géneros Allionia, Boerhavia y Mirabilis.

\begin{tabular}{|l|c|c|c|c|c|c|}
\hline \multicolumn{1}{|c|}{ Especie } & $\begin{array}{c}\text { Diámetro de los } \\
\text { granos }(\mu \mathrm{m})\end{array}$ & $\mathrm{N}^{\circ}$ de poros & $\begin{array}{c}\text { Diámetro de los } \\
\text { poros }(\mu \mathrm{m})\end{array}$ & Exina $(\mu \mathrm{m})$ & Nexina $(\mu \mathrm{m})$ & Sexina $(\mu \mathrm{m})$ \\
\hline Allionia choisyi & $65(77) 86$ & $33-57$ & $2-6$ & $4-8$ & $2,5-5$ & $1,5-3$ \\
\hline Allionia incarnata & $72(84) 100$ & $36-55$ & $3-6$ & $3-8$ & $2,5-5$ & $0,5-3$ \\
\hline Boerhavia cordobensis & $36(78) 104$ & 20 & $2-7$ & $5-8$ & $4-6$ & $1-2$ \\
\hline Boerhavia diffusa & $52(65) 90$ & $16-25$ & $3-8$ & $4,5-8$ & $3,5-5$ & $1-3$ \\
\hline Boerhavia pulchella & $75(90) 105$ & $16-17$ & $3-5$ & $6-11$ & $4-7$ & $2-4$ \\
\hline Boerhavia spicata & $70(81) 95$ & $14-20$ & $2-2,5$ & $6-8$ & $4-5$ & $2-3$ \\
\hline Mirabilis bracteosa & $86(94) 105$ & $30-45$ & $3-7$ & $4,5-5,5$ & $2,5-3$ & $2-2,5$ \\
\hline Mirabilis jalapa & $142(157) 200$ & $79-100$ & $3-8$ & $15-23$ & $10-15$ & $5-8$ \\
\hline Mirabilis ovata & $75(95) 110$ & $50-76$ & $4-10$ & $9-13$ & $6-8$ & $3-5$ \\
\hline
\end{tabular}

\section{Género Mirabilis}

Granos apolares, radiosimétricos, de tamaño grandes a muy grandes, esferoidales, pantoporados, poros subcirculares, con membrana apertural esculturada. Exina tectada equinada, microequinada y perforaciones con engrosamiento anular, distribuidas al azar. Nexina siempre mayor a la sexina.

\section{Mirabilis bracteosa (Fig. 3 K-L)}

Granos grandes a muy grandes, poros con membrana apertural con una espina. Columelas $<$ $1 \mu \mathrm{m}$ de alto.

MEB: Tectum con espinas de 1,5-2,7 $\mu \mathrm{m}$ de alto, microespinas de $\pm 0,9 \mu \mathrm{m}$ y perforaciones con engrosamiento anular de 1-3 $\mu \mathrm{m}$ de diámetro total.

\section{Mirabilis jalapa (Fig. 3 I-J)}

Granos muy grandes, poros con membrana apertural con \pm 4 espinas. Columelas $<1 \mu \mathrm{m}$ de alto.

MEB: Tectum con espinas de $\pm 2,5 \mu \mathrm{m}$ de alto, microespinas de $\pm 0,8 \mu \mathrm{m}$ y perforaciones con engrosamiento anular de 1-2,5 $\mu \mathrm{m}$ de diámetro total. Membrana apertural con 5 a 7 espinas de $1-1,5 \mu \mathrm{m}$ de alto.

\section{Mirabilis ovata (Fig. 3 M-P)}

Granos grandes a muy grandes, poros con membrana apertural con 1 espina.

MEB: Tectum con espinas de $1-2,5 \mu \mathrm{m}$ de alto, microespinas de $\pm 0,8 \mu \mathrm{m}$, y perforaciones con engrosamiento anular de $0,5-1 \mu \mathrm{m}$ de diámetro total.
Membrana apertural con 2-3 espinas ubicadas en el centro, rodeadas de gránulos de $\pm 0,6 \mu \mathrm{m}$.

\section{Género Boerhavia}

Granos apolares, radiosimétricos, de tamaño grande a muy grande, esferoidales, pantoporados, poros subcirculares, exina tectada, equinada, microequinada, perforaciones con engrosamiento anular. Elementos esculturales distribuidos al azar. Nexina de mayor espesor que la sexina.

\section{Boerhavia cordobensis (Fig. 4 A-D)}

Granos grandes a muy grandes. Poros con membrana apertural con una microespina sobre ella. Exina equinada.

MEB: Tectum con espinas de 1-4,5 $\mu \mathrm{m}$ de alto, de ápices agudos y/o romos, perforaciones anuladas de 1-2,5 $\mu \mathrm{m}$ de diámetro total. Se aprecia que la membrana apertural está ocupada por una espina de $\pm 1,5 \mu \mathrm{m}$ de alto rodeada de gránulos. Columelas de $0,5 \mu \mathrm{m}$ de alto por $\pm 1 \mu \mathrm{m}$ de ancho.

\section{Boerhavia diffusa (Fig. 4 E-H)}

Granos grandes. Poros con membrana apertural psilada o con un elemento positivo $<1 \mu \mathrm{m}$. Exina equinada y microequinada, con elementos positivos $<1 \mu \mathrm{m}$. Columelas $\leq 1 \mu \mathrm{m}$ de alto.

MEB: Tectum con espinas de 2-2,5 $\mu \mathrm{m}$ de alto, microespinas de $\pm 0,5 \mu \mathrm{m}$ y perforaciones con engrosamiento anular de 1,5-3 $\mu \mathrm{m}$ de diámetro total. Raro membranas aperturales con espinas de 1-2 $\mu \mathrm{m}$ de alto. 

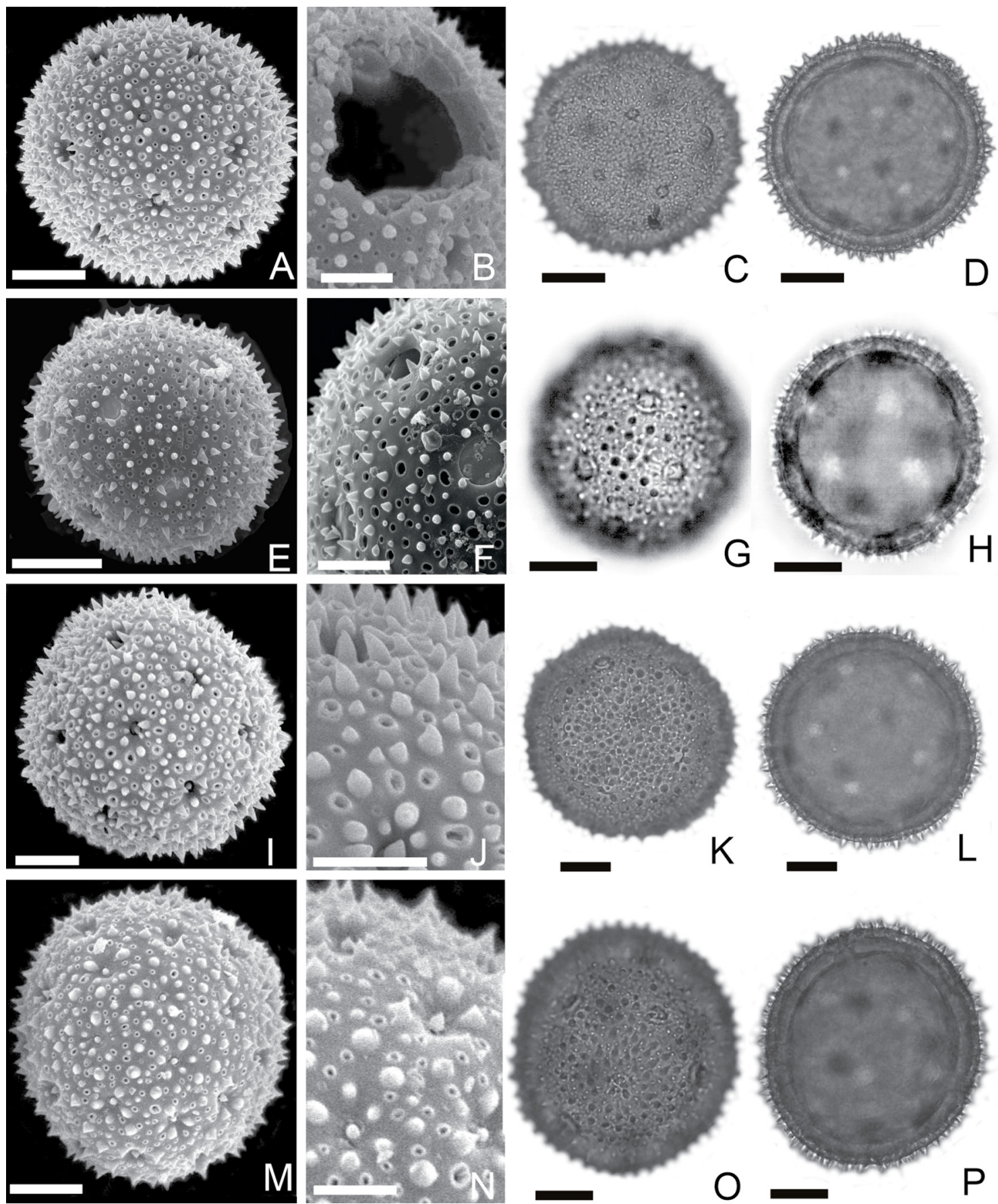

Fig. 4. Boerhavia cordobensis. A, vista general (MEB). B, detalle de la exina donde se observan las columelas bajas y perforaciones (MEB). C, vista general (MO). D, corte óptico (MO). Boerhavia diffusa. E, vista general (MEB). F, detalle de la exina y poro (MEB). G, vista general (MO). H, corte óptico (MO). Boerhavia pulchella. I, vista general (MEB). J, detalle de la exina y poro (MEB). K, vista general (MO). L, corte óptico (MO). Boerhavia spicata. M, vista general (MEB). $\mathbf{N}$, detalle de la exina y poro (MEB). $\mathbf{O}$, vista general (MO). $\mathbf{P}$, corte óptico (MO). La escala equivale a $20 \mu \mathrm{m}$ en A, C, D, E, G, H, I, K, L, M, O, P y a $5 \mu \mathrm{m}$ en B, F, J, N. 

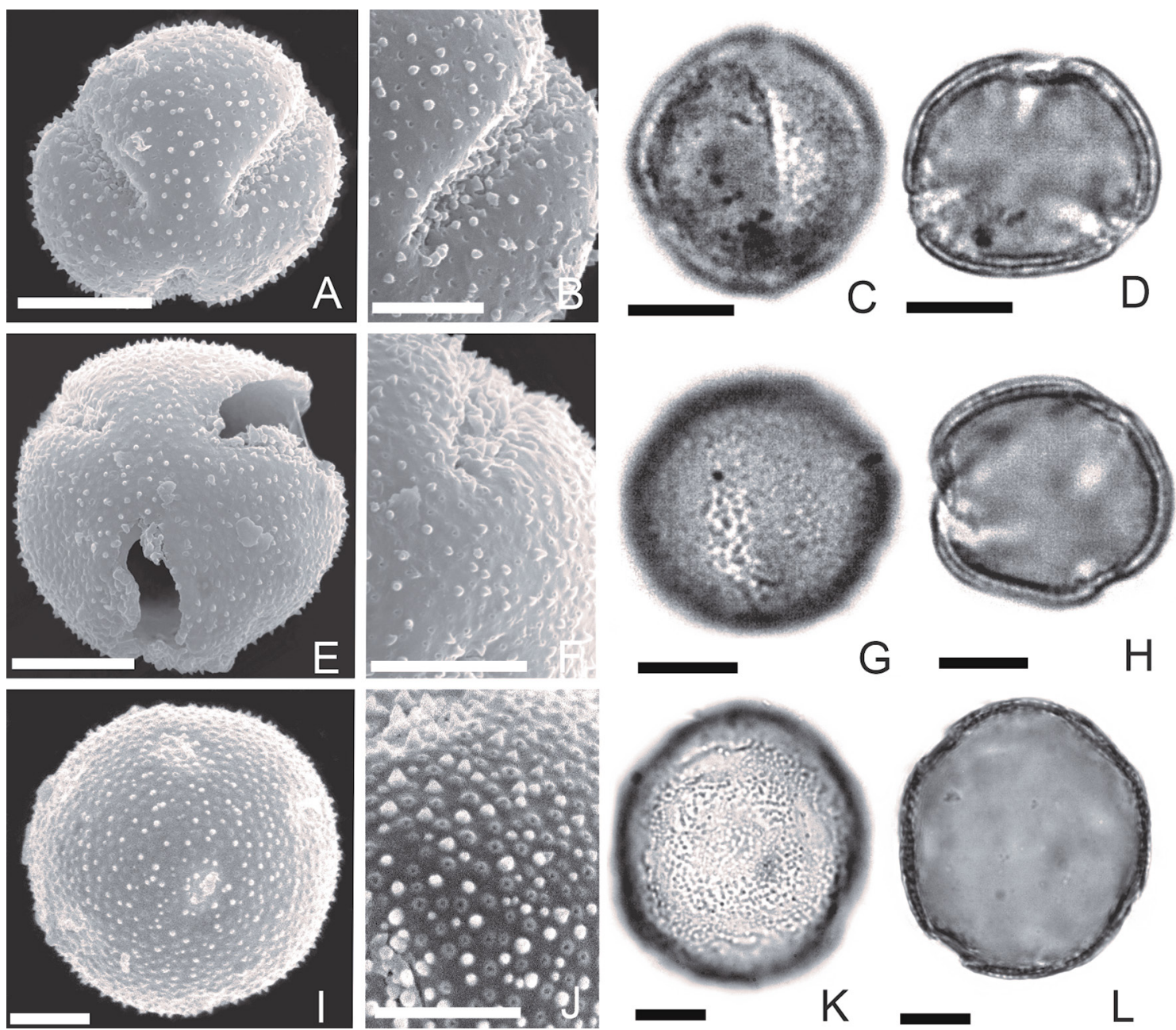

Fig. 5. Pisonia aculeata. A, vista polar (MEB). B, detalle de la exina y membrana colpal (MEB). C, vista ecuatorial (MO). D, vista polar en corte óptico (MO). Pisonia zapallo. E, vista polar (MEB). F, detalle de la exina y membrana colpal (MEB). G, vista ecuatorial (MO). H, vista polar en corte óptico (MO). Pisoniella glabrata. I, vista general (MEB). J, detalle de la exina (MEB). K, vista general (MO). L, corte óptico (MO). La escala equivale a $10 \mu \mathrm{m}$.

\section{Boerhavia pulchella (Fig. 4 I-L)}

Granos grandes a muy grandes. Poros con membrana apertural con una (raro dos) microespinas. Exina equinada y microequinada. Columelas $<1 \mu \mathrm{m}$ de alto.

MEB: Tectum con espinas de 1-3,5 $\mu \mathrm{m}$ de alto, escasas microespinas de $\pm 0,7 \mu \mathrm{m}$ y perforaciones con engrosamiento anular de 0,7-2,5 $\mu \mathrm{m}$ de diámetro total. Membrana apertural con una sola espina de $2-3 \mu \mathrm{m}$ de alto que ocupa la mayor parte del diámetro del poro.

\section{Boerhavia spicata (Fig. 4 M-P)}

Granos grandes. Poros con membrana apertural con elementos positivos bajos. Exina equinada y microequinada. Columelas $<1 \mu \mathrm{m}$ de alto.

MEB: Tectum con espinas de 1-4 $\mu \mathrm{m}$ de alto, microespinas $\pm 0,6 \mu \mathrm{m}$ y perforaciones con engrosamiento anular de $\pm 2,5 \mu \mathrm{m}$ de diámetro total. La membrana apertural está ornamentada con gránulos rodeando a una espina central de \pm $1,5 \mu \mathrm{m}$ de alto. 
Tabla 3. Valores del eje polar (P), diámetro ecuatorial(E), espesor de la exina, sexina y nexina de las especies del género Pisonia.

\begin{tabular}{|l|c|c|c|c|c|c|}
\hline \multicolumn{1}{|c|}{ Especies } & $\mathrm{P}(\mu \mathrm{m})$ & $\mathrm{E}(\mu \mathrm{m})$ & $\mathrm{P} / \mathrm{E}$ & Exina $(\mu \mathrm{m})$ & Sexina $(\mu \mathrm{m})$ & Nexina $(\mu \mathrm{m})$ \\
\hline Pisonia aculeata & $23-32$ & $21-29$ & 1,1 & $1,4-2$ & $0,7-1$ & $0,7-1$ \\
\hline Pisonia zapallo & $21-30$ & $25-30$ & 0,8 & $1-2,7$ & $0,7-2,1$ & $0,3-0,7$ \\
\hline
\end{tabular}

\section{Tribu Pisonieae (Figs. 5 y 6 ) Género Pisonia}

Granos isopolares, radiosimétricos, de tamaño mediano, de ámbito subcircular a subtriangular, prolato-esferoidales a suboblatos, tricolpados. Exina tectada con microespinas y perforaciones. Los valores del eje polar, diámetro ecuatorial, espesor de la exina, sexina y nexina de las especies del género se expresan en la Tabla 3.

\section{Pisonia aculeata (Fig. 5 A-D)}

Granos prolato-esferoidales. Colpos de 12-25 $\mu \mathrm{m}$ de largo con membrana apertural esculturada. Mesocolpio de 15-20 $\mu \mathrm{m}$ y apocolpio de 5-20 $\mu \mathrm{m}$. Columelas $<1 \mu \mathrm{m}$ de alto.

MEB: Tectum con microespinas de 0,3-0,7 $\mu \mathrm{m}$ de alto y perforaciones de 0,1-0,4 $\mu \mathrm{m}$ de diámetro. La membrana apertural del colpo está cubierta con microespinas de $\pm 0,5 \mu \mathrm{m}$ de alto, más densamente ubicadas en comparación al resto de la exina.

\section{Pisonia zapallo (Fig. 5 E-H)}

Granos sub-oblatos. Colpos de 15-24 $\mu \mathrm{m}$ de largo con membrana apertural que posee escultura similar al resto de la exina. Mesocolpio de 15-22 $\mu \mathrm{m}$ y apocolpio de 6-12 $\mu \mathrm{m}$. Columelas $<1 \mu \mathrm{m}$ de alto.

MEB: Tectum con microespinas de $\pm 0,4 \mu \mathrm{m}$ y perforaciones con engrosamiento anular de $0,2-0,5 \mu \mathrm{m}$ de diámetro total. La membrana del colpo está cubierta de microespinas de $\pm 0,8 \mu \mathrm{m}$ de alto, dispuestas en mayor densidad respecto al resto de la exina.

\section{Género Pisoniella}

Granos apolares, radiosimétricos, de tamaño mediano, esferoidales, pantocolpoidados, exina tectada con microespinas y perforaciones.

\section{Pisoniella glabrata (Fig. 5 I-L y Fig. 6 A)}

Granos de 25-55 $\mu \mathrm{m}$ de diámetro. Colpoides en número de 12 de $6-10 \mu \mathrm{m}$ de largo por 4-5 $\mu \mathrm{m}$ de ancho, describen una organización regular como si se dispusieran orientados sobre las aristas de un cubo regular imaginario (ver esquema representativo). Exina escabrada. Nexina y sexina de igual espesor con $1 \mu \mathrm{m}$ cada una. Columelas $<1 \mu \mathrm{m}$ de alto.

MEB: Tectum con microespinas de 0,5-0,9 $\mu \mathrm{m}$ y perforaciones con engrosamiento anular de $0,3-0,6 \mu \mathrm{m}$ de diámetro total. Se constata que los colpoides tienen una membrana apertural con escasas microespinas similares a las del resto de la exina.

Los ocho géneros de Nyctaginaceae de Argentina pueden diferenciarse a partir de sus granos de polen por la estructura y escultura de la exina, tipo y número de aperturas y tamaño de los granos. Es de destacar que la mayoría de los géneros (Colignonia, Pisoniella, Boerhavia, Allionia, Mirabilis y Pisonia) tiene granos con exina tectada, equinada en oposición a Bougainvillea y Reichenbachia donde es semitectada, reticulada. Las características generales de Allionia y Mirabilis son muy semejante slo que impide distinguir ambos géneros, razón por la cual en la clave están juntas.

Por otra parte, entre Bougainvillea y Reichenbachia sólo el tamaño de los granos y la presencia de lúmenes con o sin elementos libres son los únicos caracteres que permiten diferenciarlos al MO y al MEB. No obstante, en el resto de los géneros que tienen granos de polen con exina tectada, microequinada, variadas son las características que llevan a su diferenciación.

Todo ello permitió elaborar una clave palinológica para distinguir los citados géneros. 


\section{Clave polínica para el reconocimiento de granos de polen de géneros de Nyctaginaceae de Argentina.}

1. Granos de polen tectados, equinados, microequinados …........................................................ 2

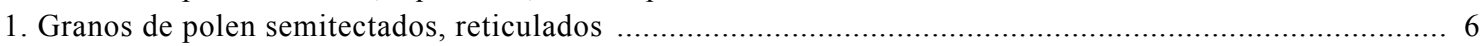

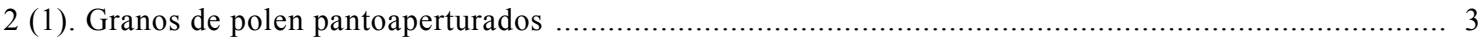

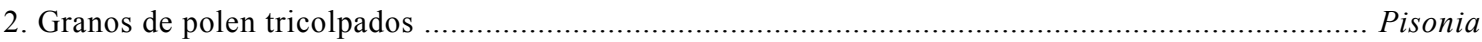

3 (2). Granos de polen de tamaño mediano …........................................................................... 4

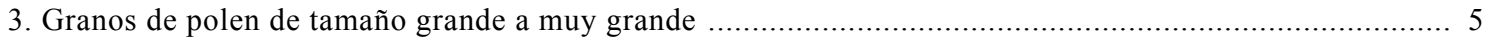

4 (3). Granos de polen pantoporados ..................................................................................... Colignonia

4. Granos de polen pantocolpoidados ................................................................................... Pisoniella

5 (3). Granos de polen pantoporados con 14 a 25 poros .......................................................... Boerhavia

5. Granos de polen pantoporados con 30 a 100 poros ...................................................... Allionia-Mirabilis

6 (1). Granos de polen de tamaño mediano y lúmenes del retículo con elementos libres ............ Bougainvillea

6. Granos de polen de tamaño pequeño y lúmenes del retículo sin elementos libres ................. Reichenbachia

Las especies de Bougainvillea no pueden diferenciarse a partir de sus granos de polen, pues sus características morfológicas son compartidas entre ellas. Dado que otros autores como Albuquerque de Souza (2010), Perveen \& Qaiser (2001), Roubik \& Moreno (1991), Nowicke (1970) llegaron a iguales conclusiones, se interpreta esta similitud interespecífica como un rasgo propio del género que impide la determinación de las especies de Bougainvillea a partir del polen.

Las especies de Allionia tampoco pueden diferenciarse entre ellas, no obstante, los granos de polen de $A$. choisyi son en general de un diámetro promedio inferior al de A. incarnata.

Los granos de polen de las especies de Boerhavia están comprendidos entre grandes y muy grandes $\mathrm{y}$ no pueden ser diferenciadas entre ellas, pues sus medidas se solapan en el espesor de la exina,
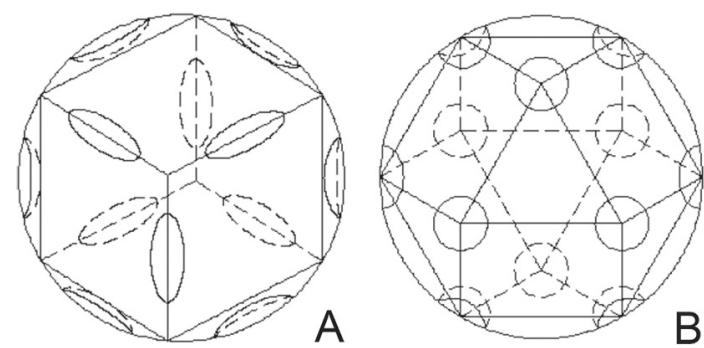

Fig. 6. Esquema representativo que muestra la distribución de las aperturas. A, Pisoniella glabrata. B, Colignonia glomerata. número de aperturas, diámetro de sus poros y en la presencia o no de escultura en la membrana apertural. No obstante $B$. cordobensis se distingue por la ornamentación equinada del tectum.

Entre las especies de Mirabilis, el número de poros es el único carácter polínico observable al MO que permite separar sus especies, pero al MEB se observan diferencias también en cuanto al tipo y número de los elementos esculturales de la membrana de los poros.

En el género Pisonia, las especies $P$. aculeata y $P$. zapallo comparten en el polen la mayoría de las características con la excepción de la forma de sus granos, constituyendo el único carácter que los diferencia.

\section{Clave polínica para diferenciar especies del género Pisonia}

1. Granos de polen subprolatos a prolatos esferoidales P. aculeata

1. Granos de polen suboblatos P. zapallo

Por último, las descripciones de los granos de polen de los géneros y especies agrupados por tribu permiten inferir que las tribus Bougainvilleeae, Colignonieae, Nyctagineae y Leucastereae pueden caracterizarse por el tipo polínico, señalado por el tipo de apertura, forma, tamaño, polaridad y exina, con la excepción de Pisonieae que reúne la conjunción de caracteres presentes en las otras tribus (Tabla 4). 
Tabla 4. Relaciones comparativas entre las tribus de Nyctaginaceae de Argentina.

\begin{tabular}{|c|c|c|c|c|c|}
\hline Tribu & Polaridad & Forma & Tamaño & Apertura & Exina \\
\hline Bougainvilleeae & Isopolar & $\begin{array}{c}\text { Oblato-esferoidal,Sub- } \\
\text { oblato }\end{array}$ & Mediano & Tricolpados & $\begin{array}{c}\text { Semitectada, } \\
\text { reticulada }\end{array}$ \\
\hline Colignonieae & Apolar & Esferoidal & Mediano & Pantoporados (12 poros) & $\begin{array}{c}\text { Tectada, } \\
\text { microperforada }\end{array}$ \\
\hline Nyctagineae & Apolar & Esferoidal & $\begin{array}{c}\text { Grande a } \\
\text { muy grande }\end{array}$ & Pantoporados (14-100 poros) & Tectada, perforada \\
\hline Leucastereae & Isopolar & Oblato-esferoidal & Pequeño & Tricolpados & $\begin{array}{c}\text { Semitectada, } \\
\text { reticulada }\end{array}$ \\
\hline Pisonieae & $\begin{array}{c}\text { Apolar, } \\
\text { Isopolar }\end{array}$ & $\begin{array}{c}\text { Esferoidal, Oblato- } \\
\text { esferoidal, Sub-oblato }\end{array}$ & Mediano & Tricolpados, Pantocolpoidados & Tectada, perforada \\
\hline
\end{tabular}

\section{DISCUSIÓN Y CONCLUSIONES}

Considerando todas las especies nativas de Nyctaginaceae de Argentina, se brinda aquí por primera vez las características palinológicas de Allionia choisyi, Boerhavia cordobensis y Boerhavia spicata, las cuales no cuentan con antecedentes publicados en el tema.

Los estudios palinológicos de las Nyctaginaceae argentinas son escasos. Caccavari (1979) publicó 9 géneros, 16 especies y 3 variedades con descripciones e ilustraciones sólo al MO. De su comparación con nuestras descripciones, se evidencian coincidencias en la generalidad de sus caracteres, con la excepción de los de A. incarnata; $B$. pulchella y $M$. jalapa que registran un menor diámetro $(68,68$ y 147 $\mu \mathrm{m}$ respectivamente); $M$. bracteosa con mayor diámetro $( \pm 119 \mu \mathrm{m})$ y espesor de la exina $( \pm$ $9 \mu \mathrm{m}) ;$ M. ovata (descrita dentro del género Oxybaphus L'Hérit. ex Willd., O. ovatus) con un número menor de poros (44) y $B$. diffusa (bajo el epíteto específico $B$. coccinea). Las especies $B$. campanulata; $B$. infesta; $B$. praecox; $B$. spinosa; B. stipitata; P. aculeata; P. zapallo y $R$. paraguayensis, discrepan por el mayor tamaño, la forma de sus granos y la presencia de microespinas supratectales visibles al MO. Estas diferencias son atribuidas a que las observaciones y mediciones efectuadas al MEB aportan mayor precisión y detalle en las descripciones.

Para Brasil meridional, Barth \& Ferreira Barbosa (1972) describen mediante MO y con ilustraciones muy detalladas, el polen de las plantas arbóreas de la región, entre ellas incluyen el de $M$. jalapa con un diámetro y elementos esculturales ligeramente mayores a los nuestros, también el de $B$. coccinea (sinonimizada a $B$. diffusa por Standley \& Steyermark, 1946) con exina y elementos esculturales mayores (espinas de $\pm 6 \mu \mathrm{m}$ de alto). Por último, $P$. aculeata con polen de forma esferoidal y exina de mayor espesor a los de aquí estudiados.

Albuquerque de Souza et al. (2010) analizan para Río de Janeiro (Brasil), los granos de polen de B. diffusa y M. jalapa. La primera de ellas una con exina de mayor espesor $( \pm 12 \mu \mathrm{m})$ respecto a la de aquí analizada, que alcanza hasta $\operatorname{los} 8 \mu \mathrm{m}$, donde la nexina y sexina son iguales. Es de destacar que, contrariamente a lo señalado, en los granos de polen que integran la tribu Nyctagineae (incluida B. diffusa), la nexina es siempre mayor a la sexina. M. jalapa, por su parte, es descripta con un número de poros menor (32 poros) mientras que el resto de los caracteres palinológicos coinciden con los rangos hallados.

Nowicke (1970), también describe al MO a $M$. jalapa con características muy semejantes a las del presente trabajo. Nowicke \& Luikart (1971) analizan con MEB a Reichenbachia hirsuta (sinonimizada a $R$. paraguayensis por Zuloaga \& Morrone, 1999) difiriendo en la forma, tamaño y presencia de elementos supratectales. Perveen \& Qaiser (2001) en la flora polínica para Pakistán y Rao \& Leong (1974) en la morfología polínica de plantas tropicales, describen a los granos de polen de $B$. diffusa con las mismas características aquí observadas. 
De lo analizado se desprende que las descripciones palinológicas de las especies $B$. diffusa y $M$. jalapa son las que aparecen en la mayoría de los trabajos (Nowicke, 1970; Nowicke \& Luikart, 1971; Barth \& Ferreira Barbosa, 1972; Rao \& Leong, 1974; Caccavari, 1979; Del Pino \& Díez, 1990; Perveen \& Qaiser, 2001; Albuquerque de Sousa et al., 2010), y que a su vez presentan mayor variabilidad en los parámetros de los caracteres palinológicos.

Por otra parte, es importante destacar que López et al. (2006) analizaron la relación entre distintas variables, como el tamaño de los granos de polen y el tipo de reserva alimenticia (lípidos y almidón) de 14 especies de Nyctaginaceae de Argentina. En ella encontraron que los granos de polen más grandes, pertenecientes a las especies de los géneros Allionia, Boerhavia y Mirabilis (Tribu Nyctagineae) están directamente relacionados con la reserva de almidón, mientras que los más pequeños, como los de Bougainvillea (Tribu Bougainvilleeae), con la de lípidos.

Para América del Norte, Douglas \& Manos (2007) proporcionan un examen exhaustivo a nivel de género de la filogenia de las Nyctaginaceae, y construyen un árbol filogenético a partir de secuencias de tres loci cloroplásticos, señalando que la tribu Nyctagineae y sus subtribus, propuestas por Bittrich \& Kühn, 1993 en Douglas \& Manos 2007 son parafiléticas, debido a la excesiva dependencia de algunos caracteres homoplásicos como son la morfología del polen y la presencia del involucro en la flor. Douglas \& Spellenberg (2010), retoman dicho trabajo y caracterizan palinológicamente las tribus de Nyctaginaceae del mundo (Bougainvilleeae, Colignonieae, Leucastereae, Nyctagineae, Pisonieae, Boldoeae y Caribeeae), respecto de los géneros representados en Argentina, la filogenia de estos autores propone que Allionia, Mirabilis y Boerhavia se incluyen en la tribu Nyctagineae, separando a Colignonia (antiguamente incluida en esta tribu en la clasificación de Bittrich \& Kühn, 1993), con Allionia y Mirabilis en un grupo hermano al de Boerhavia; por otro lado agrupan a los géneros Pisonia y Pisoniella en la tribu Pisonieae, con Pisoniella como grupo hermano al resto de los géneros.
Respecto a estos resultados en este trabajo podemos dar apoyo a esa clasificación molecular a partir del polen, dado que las características de los géneros Allionia y Mirabilis los hacen indiferenciables entre sí, pero se pueden distinguir del género Boerhavia, dentro de la misma tribu. Colignonia posee una combinación de características palinológicas no compartidas con otras especies (constancia del número de poros, ubicación regular de los mismos y exina escabrada, con microespinas menores $0,5 \mu \mathrm{m}$ ) lo que hace posible separarlo del resto. Por último, Pisonia y Pisoniella comparten la estructura y escultura de la exina y aperturas más largas que anchas, pero las características de Pisoniella, que es esferoidal y posee colpoides, pueden explicar su posición dentro de la filogenia. Douglas \& Spellenberg (2010) también dan características morfológicas de cada tribu, entre ellas establecen algunos rasgos polínicos: granos de polen tricolpado con exina reticulada o pantocolpado para la tribu Bougainvilleeae; granos 12-pantoporados, de 17$30 \mu \mathrm{m}$ y exina espinulosa en Colignonieae; granos de polen de 100-200 $\mu \mathrm{m}$ pantoporados, con exina espinulosa o tricolpados con exina reticulada para Nyctagineae; granos 3-colpados, de 20-35 $\mu \mathrm{m}$, con exina reticulada o equinada para Leucastereae y granos generalmente tricolpados equinados en la tribu Pisonieae.

Las especies argentinas, concuerdan ampliamente con las características identificadas por los citados autores y aparecen resumidas en la Tabla 4, donde se agregan características para las tribus basadas solo en las especies nativas del país, y se amplía la descripción de la tribu Pisonieae con granos con colpoides.

Para concluir, se confirma el carácter euripalínico para las especies nativas de Nyctaginaceae argentinas establecido por Caccavari (1979). La variabilidad palinológica encontrada a nivel de género permite diferenciarlos a partir de una clave con la excepción de Allionia y Mirabilis. No obstante ello, en los géneros representados con más de una especie (Allionia, Boerhavia, Bougainvillea, Mirabilis y Pisonia) no pueden diferenciarse palinológicamente las especies entre sí (con la excepción de Pisonia y Mirabilis), por la homogeneidad y superposición de sus caracteres. 


\section{AGRADECIMIENTOS}

Deseamos expresar nuestro agradecimiento a las autoridades del Centro de Ecología del Litoral (CECOAL-CONICET-UNNE) por brindarnos las instalaciones adecuadas para la realización de este trabajo, al Instituto de Botánica del Nordeste (IBONE), quienes permitieron el acceso al herbario y a la biblioteca. A Yanina Horn (técnica de laboratorio) por la preparación del material polínico. El presente trabajo se realizó bajo el marco del Proyecto Paleovegetación y Paleoambiente de la Formación Palo Pintado, Neógeno del Norte de Argentina. Res. 970/16. (PI 16F008) Universidad Nacional del Nordeste.

\section{BIBLIOGRAFÍA}

Albuquerque de Souza, M.; C. B. Ferreira Mendoça \& F. G. Gonçalves-Esteves. 2010. Palinología de espécies de Nyctaginaceae Juss. ocurrentes nas restingas do estado do Rio de Janeiro, Brasil. Acta Botanica Brasilica 24: 104-110.

APG IV. 2016. An update of the Angiosperm Phylogeny Group classification for the orders and families of flowering plants: APG IV. Botanical Journal of the Linnean Society 181: 1-20.

Barth, O. M. \& A. F. Ferreira Barbosa. 1972. Catálogo sistemático do pólens das plantas arbóreas do Brasil. XIV Nyctaginaceae y Phytolaccaceae. Memórias do Instituto Oswaldo Cruz 70: 240-259.

Bittrich, V. \& U. Kühn. 1993. Nyctaginaceae, en Kubitzkijens, K.; G. Rohwer \& V. Bittrich (eds.), Flowering plants. Dicotyledons: Magnoliid, Hamamelid and Caryophillid families. Volumen 2, pp. 473-486. Springer, Berlín, Heidelberg.

Caccavari, M. A. 1979. Granos de polen de Nictagináceas Argentina. Revista del Museo Argentino de Ciencias Naturales "Bernardino Rivadavia" e Instituto Nacional de Investigación de las Ciencias Naturales. Botánica 5: 211-234.

Del Pino, J. A. \& M. J. Díez. 1990. Aportación a la palinología de las plantas ornamentales de la ciudad de Sevilla. I. Lagascalia 15: 199-221.

Douglas, N. A. \& P. S. Manos. 2007. Molecular phylogeny of Nyctaginaceae: taxonomy, biogeography, and characters associated with a radiation of xerophytic genera in North America. American Journal of Botany 94: 856-872.
Douglas, N. \& R. Spellenberg. 2010. A new tribal classification of Nyctaginaceae. Taxon 59: 905-910.

Erdtman, G. 1966. Pollen morphology and Plant Taxonomy Angiosperms. Hafner Publishing Company. New York. 553p.

Erdtman, G. \& H. Straka. 1961. Cormophyte spore classification. An outline based on the aperture (Tremata). Forhandlingar; Geologiska Foreningeni Stockholm. 83: 65-78.

Farrell, E. E. 2017. Morfología polínica de las especies de Nyctaginaceae Juss. en Argentina y su aplicación al registro fósil. Tesis de pregrado. Universidad Nacional del Nordeste. Corrientes, Argentina.

Farrell, E. E. \& L. R. Mautino. 2018. Estudio palinológico de especies de Nyctaginaceae Juss. Nativas de Argentina. Simposio de Paleobotánica y Palinología, Paraná, Argentina: 68.

Kannabiran, B. 1973. Pollen morphology in Boerhavia punarnava Saha \& Krish. Science Culture 39: 280-281.

Kremp, G. O. W. 1968. Morphologic Encyclopedia of Palynology. The University of Arizona Press. Tucson, Arizona. 263p.

López, H. A.; A. M. Anton \& L. Galetto. 2006. Pollen-pistil correlation and pollen size-number trade-off in species of Argentinian Nyctaginaceae with different pollen reserves. Plant Systematics and Evolution 256: 69-73.

Mabberley, D. J. 1987. The Plant Book. Cambridge University Press. Cambridge. 707 p.

Markgraf, V. \& H. D’Antoni. 1978. Pollen Flora of Argentina. Modern Spore and Pollen. Types of Pteridophyta, Gymnospermae and Angiospermae. The University of Arizona Press. Tucson. Arizona. 208p.

Nilsson, S. \& J. Praglowski. 1992. Erdtman's Handbook of Palynology. 2da. ed. Munksgaard, Copenhagen. 582p.

Nowicke, J. W. 1970. Pollen morphology in the Nyctaginaceae. Grana 10: 79-88.

Nowicke, J. W. \& T. J. Luikart. 1971. Pollen morphology of the Nyctaginaceae. Grana 11: 145-150.

Perveen, A. \& M. Qaiser. 2001. Pollen flora of Pakistan -XXVII Nyctaginaceae. Turkish Journal of Botany 25: 385-388.

Punt, W.; P. P. Hoen; S. Blackmore; S. Nilsson \& A. Le Thomas. 2007. Glossary of pollen and spore terminology. Review of Palaeobotany and Palynology 143: 1-81.

Ramírez Arriaga, E.; A. Martínez Bernal; N. Ramirez Maldonado \& E. Martínez Hernández. 2016. Análisis palinológico de mieles y cargas de polen de Apis mellifera (Apidae) de la región Centro y Norte del estado de Guerrero, México. Botanical Sciences 94: 141-156.

Rao, A. N. \& Leong, F. L. 1974. Pollen Morphology of Certain Tropical Plants. Reinwardtia 9: 153-176. 
Rodríguez de la Cruz, D.; E. Sánchez Reyes; S. Sánchez Durán \& J. Sánchez Sánchez. 2013. Análisis palinológico de mieles comerciales monoflorales. Botanica Complutensis 37: 171-180.

Roubik, D. W. \& J. E. Moreno. 1991. Pollen and spores of Barro Colorado Island [Panamá]. Monographs in Systematic Botany from the Missouri Botanical Garden. 36.

Sáenz Laín, C. 2004. Glosario de términos palinológicos. Lazaroa 25: 93-112.

Sánchez-Dzib, Y. A.; S. Sosa-Nájera \& M. S. LozanoGarcía. 2009. Morfología polínica de especies de la selva mediana subperennifolia en la cuenca del río Candelaria, Campeche. Boletín de la sociedad botánica de México 84: 83-104.

Standley, P. C. \& J. A. Steyermark. 1946. Nyctaginaceae. En: Standley, P. C. \& J. A. Steyermark. (Eds). Flora of Guatemala- part IV. Fieldiana. Botany López 24: 174-192.
Tripathi, S.; S. Singh \& R. K. Roy. 2017. Morfología del polen de buganvillas (Nyctaginaceae): una planta ornamental popular de los jardines tropicales y subtropicales del mundo. Revisión de Paleobotánica y Palinología 239: 31-46.

Xu, Z. \& M. Deng. 2017. Nyctaginaceae, en Identification and Control of Common Weeds: Volume 2, pp. 303-308. Springer, Dordrecht.

Zuloaga, F. O. y O. Morrone. 1999. Catálogo de las Plantas Vasculares de la República Argentina II. Monographs in Systematic Botany from the Missouri Botanical Garden 74 (F-Z) 623-1267.

Zuloaga, F. O.; O. Morrone \& M. J. Belgrano (eds.). 2008. Catálogo de las Plantas Vasculares del Cono Sur (Argentina, Sur de Brasil, Chile, Paraguay y Uruguay). Monographs in Systematic Botany from the Missouri Botanical Garden 107: 985-2286. 